\title{
Influence of Chemical Admixtures and Environmental Conditions on Initial Hydration of Concrete
}

\author{
A. Bettencourt Ribeiro ${ }^{1}$, V. Aguiar Medina ${ }^{2}$ and A. Martins Gomes ${ }^{2}$ \\ ${ }^{1}$ LNEC-Portuguese National Laboratory of Civil Engineering \\ ${ }^{2}$ Instituto Superior Técnico, Technical University of Lisbon
}

\begin{abstract}
There has been an increasing number of problems in concrete pavements, such as cracks and delamination, in which surface hardeners have been used, especially in car parks, industrial areas, warehouses etc. Factors that can potentiate the occurrence of these problems are the increasing use of more effective water reducers in concrete and also the use of binders with higher fineness and higher tricalcium silicate content than the former ones. Concrete and surface hardeners are both cementitious materials, which however are applied at different instants. Distinct stages of hydration may lead to lack of homogeneity and, eventually, to cracking and delamination. This study evaluates early hydration and bleeding behaviour of concrete, which are relevant factors for finishing with surface hardeners. The influence of chemical admixtures and environmental conditions were tested by penetration resistance and bleed water, which highlights the shortening of the period in which surface hardener application is possible.
\end{abstract}

\section{Introduction}

A significant increase in problems occurring on concrete floors has been detected, concerning pavements in which surface hardeners were used, especially in car parks, industrial areas, warehouses, etc. The negative effect of these abnormal situations has important economic implications, since the necessary work to correct them involves large areas of reconstruction and the use of expensive repairing materials, and also because it often delays the completion of the construction work and, hence the beginning of the infrastructure operation. There is no systematic survey on the number of works in which this the problem occurs, but LNEC (National Lab. of Civil Eng.) has been asked to intervene in many cases of cracking and detachment of the concrete surface, in various regions of Portugal and for different entities, which shows that is a widespread problem, rather than just local or circumscribed [1-2]. The application of surface hardeners to pavements is 